\title{
Experience in the Treatment of Choledochal Cyst with Robot-Assisted Surgery in Children: and "The Current State of Minimally Invasive Surgery in this Anomaly"
}

\section{Mario Navarrete Arellano*}

Paediatric Surgery, Hospital Central Militar, SEDENA, Mexico City, Mexico

*Corresponding Author: Mario Navarrete Arellano, Paediatric Surgery, Hospital Central Militar, SEDENA, Mexico City, Mexico.

Received: August 13, 2019; Published: October 05, 2019

\begin{abstract}
Objective: Report our experience in the treatment of choledochal cyst with robot-assisted surgery in children and perform a current analysis of both approaches of minimally invasive surgery, for this anomaly.

Material and Methods: Study observational, prospective, and longitudinal, in pediatric patients with choledochal cyst treated with robotic-assisted surgery, from Jul-2016 to Apr-2019. The diagnosis was made with laboratory studies, USG and cholangioresonance. The surgery performing one part by laparoscopy, the extracorporeal Roux-en-Y, and other part with robotic assistance (cholecystectomy, retrocolic Roux-en-Y, and the hepaticojejunostomy). We use 5 trocars, 4 robotics and a laparoscopic. Registered variables: demographic data, cysts according to Todani's classification, size, clinic, total and robotic surgery time, bleeding, conversions, complications, postoperative stay and follow-up. We carry out a detailed non-systematic review of previous publications on this pathology with conventional laparoscopic and robotic surgery.

Results: We treated 4 patients with choledochal cyst, relation 1:3 female, the averages was in age 3 years, weight $15 \mathrm{~kg}$ and height $94.2 \mathrm{~cm}$. Type 1-A cysts were three and one 1-C, the average size of the cysts, $9.6 \mathrm{~cm}$. Clinically, they presented: abdominal mass 3 , jaundice episode 3 and fever 2 . The following are average values of: total surgical times $360 \mathrm{~min}$ and console surgery time $230 \mathrm{~min}$, bleeding $32.5 \mathrm{ml}$, and postoperative stay 4.7 days. Conversions, complications and mortality 0\%. Follow-up average 29 months, and all patients are asymptomatic. Currently, the number of children with choledochal cyst treated with robotic surgery, barely reaches about $3 \%$, in relation to those treated with conventional laparoscopy. The initial experience was published with laparoscopy in Oct1995 and with robotic surgery in Apr-2006.

Conclusions: Our results are encouraging, and show the advantages of robotic surgery, although our experience is limited to a few cases. Robotic surgery for the treatment of choledochal cyst is feasible and safe. The current results with both minimally invasive approaches are very similar, but we consider that it represents an important bias, the great advantage in the application that conventional laparoscopy takes to robotic surgery. To date, very few pediatric surgeons in the world have applied robotic surgery to the choledochal cyst.

Keywords: Choledochal Cyst; Biliary Cyst; Biliary Tract Anomalies; Robotic Surgery; Robot-Assisted Surgery; Laparoscopy; Laparoscopic Surgery; Minimally Invasive Surgery; Hepaticojejunostomy; Extracorporeal Roux-en-Y; Children
\end{abstract}

\section{Introduction}

A choledochal cyst is a rare congenital cystic dilatation of the extra or intrahepatic biliary tract or both. It was first described by Vater and Ezler in 1723 [1].

Choledochal cysts have an incidence of 1 in 100,000-150,000 live births in the western population, but reported to be as high as 1 in 13,500 live births in the United States and 1 in 15,000 in Australia. The incidence is higher in Asian population with an incidence of 1 in 1000, of which about two-third cases are reported from Japan. Choledochal cysts are diagnosed in childhood about $75 \%$, also have an unexplained female:male preponderance, commonly reported as $4: 1$ to $3: 1$ [2].
They are classified according to the location of biliary duct dilation as described by Todani., et al. [3].

With the development and routine use of prenatal ultrasound screening, more neonates are initially diagnosed with choledochal cyst prenatally. Prenatal ultrasound diagnosis can be established or suspected, especially in the third trimester of pregnancy. With the development of imaging techniques and the prenatal screening, an increasing number of asymptomatic cases have been diagnosed [4].

Clinically, the classic triad of pain, jaundice and mass in the upper right quadrant of the abdomen is not common in children with 
choledochal cysts, it is more likely that they only present two of the three signs or symptoms. Cyst rupture is rare and is usually seen in neonates and infants. In neonates, biliary fibrosis and cirrhosis can occur due to biliary tree obstruction [5].

It is essential since it leads to early surgery with less risk of postnatal complications such as cholestasis, cholangitis, stone formation, biliary obstruction, perforation, portal vein thrombosis, biliary cirrhosis, pancreatitis, portal hypertension, liver failure, hepatic abscess, and cholangiocarcinoma [6].

Current surgical management ideal evolved primary cyst excision with Roux-en-Y bilioenteric drainage, and furthermore, a few type IVA and type $\mathrm{V}$ choledochal cyst patients may need hepatic resection or liver transplantation [2].

In relation to reconstruction after a complete resection of the choledochal cyst, the most commonly used alternative is Roux-en-Y with hepaticojejunostomy, and the other option is hepaticoduodenostomy, but the risk is bilious reflux gastritis $[2,7]$.

In 1995, Farello., et al. they report first experience with a laparoscopic treatment of congenital choledochal cysts involving the total cyst resection and the reconstruction of the biliary and gastrointestinal tracts through a transmesocolic hepatic-jejunal Rouxen-Y loop anastomosis [8].

In 2012, was reported for the first time single-port transumbilical laparoscopic surgery of the hepaticojejunostomy with Roux-enY in children with choledochal cyst and had a satisfactory outcome. But, reports of its application in the pediatric population are limited, because the technique requires experienced hands. However, the technique is reproducible and has become the main surgical treatment for choledochal cyst in China [9].

Laparoscopic resection of choledochal cyst has been widely used, but its early complications occurred frequently and should be given more attention. The postoperative complications related to the surgical technique are: anastomotic leaks, biliary fistulas, stenosis, Roux-en-Y obstruction and colangitis, duodenal obstruction, pancreatitis, pancreatic fistula, cirrhosis and liver failure [10].

Over the last two decades, with the advent of laparoscopy, several authors have reported the feasibility and benefits of laparoscopic chodedochal cyst excision [11].

They argue the advantage of obtaining excellent visualization of the portal structures with laparoscopic surgery [12], other advantages are: minimal scarring, less pain, less bleeding and transfusions, less complications, less risk of infection, faster recovery and soon return to daily activities. But there are also inconveniences, the child has an abdominal cavity small, poor tolerance of the pneumoperitoneum, in addition to the need for an anastomosis bilio-digestive accurate, are aspects that are challenging, and the surgeon requires a long time to acquire the skills and confidence enough, in complex techniques like this, versus with robot-assisted surgery, the learning curve is shorter. However, the laparoscopic approach it has not been widely used, because especially the laparoscopic hepaticojejunostomy are quite technically demanding [13]. Although this approach is safe and effective in the hands of experts [14], and also in hospitals with a high volume of cases.

For the first time, the robot-assisted laparoscopic minimally invasive approach to choledochal cyst was performed by Woo., et al. in 2006. He used robotic assistance to facilitate the minimally invasive treatment of a type I choledochal cyst in a girl of 5-year-old. They concluded that resection appears safe and feasible, the threedimensional visualization and wristed instrumentation greatly aids in the dissection of the cyst and in the biliary reconstruction. Robotic enhancements offer improvements to conventional minimal access surgery, permitting technical capabilities beyond existing threshold limits of human performance for surgery within the spatially constrained operative workspaces in children, the camera controlled by the primary surgeon, intuitive instrument control, tremor reduction, and, most importantly, enhanced dexterity and mobility of the wristed instruments that allow precise dissection and anastomosis [15]. The above are advantages for the surgeon, which benefit the patient.

The current alternatives are, open surgery, laparoscopy conventional and robotic surgery for treatment of the choledochal cyst.

In our experience, hepaticojejunostomy has been the reconstruction alternative after resection of the choledochal cyst, first with open surgery and then directly jump to robotic surgery, due to the limitations of conventional laparoscopy, since our casuistry is scarce.

The objective is report our experience in the treatment of choledochal cyst with robot-assisted surgery in children, and perform a current analysis of the approaches minimally invasives: laparoscopic conventional and robotic surgery in this pathology.

\section{Materials and Methods}

The study is observational, prospective, and longitudinal, in pediatric patients with choledochal cyst treated with robotic-assisted laparoscopic surgery, the sample was conventional, they were included in all patients undergoing this treatment from Jul-2016 to Apr-2019. Our hospital is a public tertiary care facility.

The diagnosis was made with laboratory studies, USG and cholangioresonance (Figure 1). Registred variables: demographic, type 
Experience in the Treatment of Choledochal Cyst with Robot-Assisted Surgery in Children: and "The Current State of Minimally Invasive Surgery in this Anomaly"

of choledochal cyst according to Todani's classification, size of the cyst, clinical picture, total time of surgery and robotic time, bleeding, conversions, complications, hospital stay postoperative and follow-up. The patients during the transoperative period were only managed with two peripheral venous channels, nasogastric tube and urinary catheter and non-invasive monitoring.


Figure 1: Ultrasound and colangioresonance images, cases 1 to 3 , type 1 -A choledochal cysts and case 4 type $1-\mathrm{C}$.

Five ports were used: robotic of $8.5 \mathrm{~mm}$ for $30^{\circ}$ len, for 3D-HD image, 3 for robotic instruments of $8 \mathrm{~mm}$ and a $5 \mathrm{~mm}$ laparoscopic for an Assistant. Patient position in inverted trendelemburg with elevation of $30^{\circ}$, before doing the docking (Figure 2). The docking charts for robotic surgery that are suggested for surgical techniques in adults were not applicable for children. Thus, at times, $3 \mathrm{~cm}$ of separation was possible between each trocar when performing surgery on infants, due to the limited space in such a small patient.

The surgery for minimal invasion, performing one part assisted by laparoscopy, the extracorporeal Roux-en-Y and the other part with robotic assistance (resection of the cyst and gallbladder, the retrocolic step of the Roux-en-Y and the hepaticojejunostomy). Between 15 and $20 \mathrm{~cm}$ below the Treitz angle, the jejunal loop was externalized by wound in the umbilical scar to do the extracorporeal Roux-en-Y and jejunal-jejunal anastomosis, regardless of whether the port for the camera was placed at this point (Figure 3).
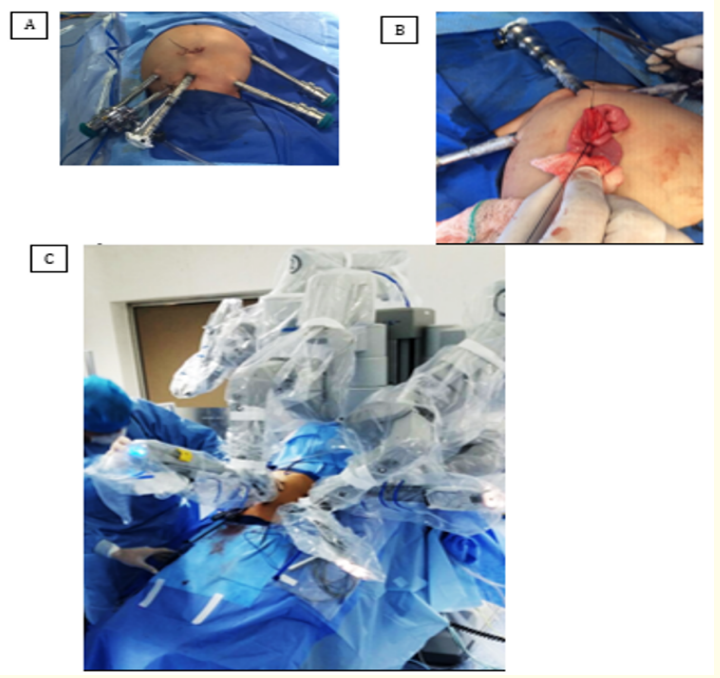

Figure 2: A. Location of the 5 trocars, robotics 4: camera lens and 3 working, and the laparoscopic or auxillary. B. Umbilical scar wound and extracorporeal Roux-en-Y. C. Inverted trendelenburg position and docking.
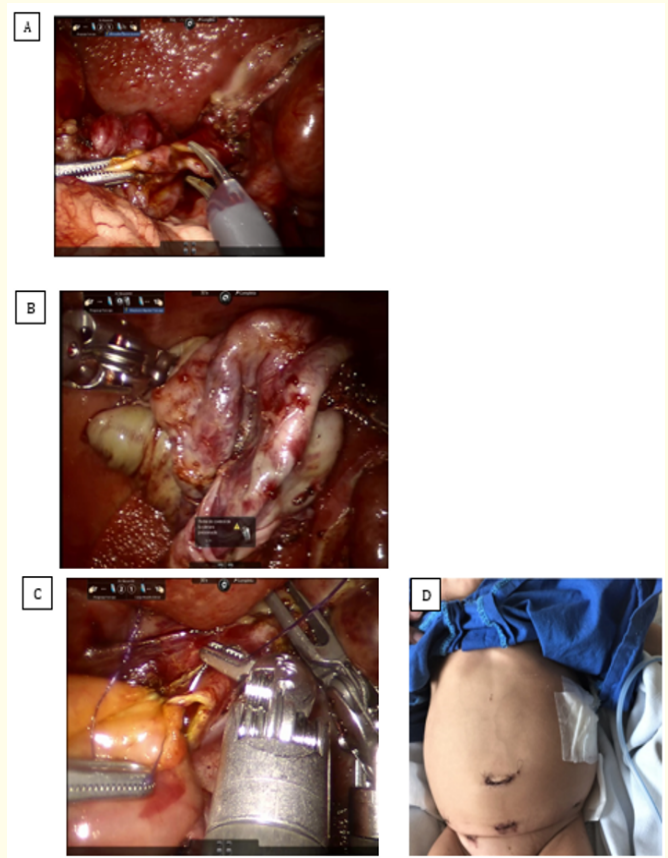

Figure 3: Case 2. Transoperative images of robotic surgery, A. Complete dissection of the choledochal cyst and cut at the level of the common hepatic duct. B. Surgical piece already separated. C. Hepatic-jejunal anastomosis, articulated robotic instruments with 7 freedoms of movement, easy intracorporeal suture. D. Case 2, abdomen on the postoperative 3rd day, receiving normal food. 
The biliodigestive anastomosis was performed with absorbable suture PDS 4-0, continuous suture in 2 parts, posterior and anterior lip, a single plane. At the end of the surgery, an active drainage is left in the surgical bed, leaving it by the wound of the trocar 3 robot located in the mid-axillary left subcostal line, which was withdrawn on the day of discharge (Figure 2).

The Clavien-Dindo classification of surgical complications was used [16]. The surgical system used was the da Vinci model, Si version (Intuitive Surgical, Inc., Sunnyvale, CA. U.S.A).

It was protocolized start in the postoperative period feeding the patients when they restored his intestinal transit, previous retirement of the nasogastric tube.

The postoperative follow-up was at 8,30 , and 90 to 120 days, and then every 6 months. It was previously defined for this pathology that during the follow-up postoperative, laboratory, radiographic and/or cabinet studies were carried out, only if the patients present symptoms or signs of possible complications, to evaluate the results.

The data was entered into a spreadsheet in Microsoft Office Excel 2013 version.

For the analysis of the results, measures of central tendency were used.

In relation to ethical considerations of the study, being of an observational nature, it was not necessary to consent to enter the study to the patients. The Research Ethics Committee of the Hospital evaluated and approved the study. In Mexico, robot assisted surgery complies with the records and regulations of the Mexican health authorities. In our institution, robotic surgery is routinely authorized for execution. In order to perform the medical-surgical procedures, we obtained the informed consent in writing from the parents or guardians.
To evaluate the current state of minimally invasive surgery in choledococyst cyst in children, we performed a non-systematic review of previous publications in the PubMed database using the keywords: robot-assisted or robotic surgery + choledochal cysts + children.

Other searched using the keywords: choledochal cyst + laparoscopic or laparoscopic surgery + children. Then we analyze the information obtained.

\section{Results and Discussion}

Results

In the period of 34 months, four patients was treated of choledochal cyst, 3 females and 1 male (3:1 ratio), with average age of 36 months, range 6 to 58 months, average weight of $15 \mathrm{~kg}$, range 5.9 to $19.8 \mathrm{~kg}$, and average height of $94.2 \mathrm{~cm}$, range 65 to $110 \mathrm{~cm}$. they are part of the statistics of a previous publication (17).

There were three cases of cyst type 1-A and one type 1-C; the size of the cyst was $8-12.5 \mathrm{~cm}$, with an average of $9.6 \mathrm{~cm}$. Clinically, 3 cases presented with an abdominal mass, 3 cases with an episode of jaundice, and 2 with fever. In no patient was carried out prenatal diagnosis.

The average surgical times were $360 \mathrm{~min}$ total time, with range of 344 to $382 \mathrm{~min}$ and $230 \mathrm{~min}$ for console surgery time, with range of 184 to $262 \mathrm{~min}$. The average of $32.5 \mathrm{ml}$ of bleeding, range 20 to $50 \mathrm{ml}$. Index of conversions, complications and mortality $0 \%$. In the younger infant case and the larger choledochal cyst, we began surgery by partial drainage by puncture of the cyst through an avascular window of the mesentery by a wound in the umbilical scar and thus be able to have space to place the trocars and perform minimally invasive surgery.

All the patients evolved stable in the postoperative period, they were only managed with a venoclysis, the day after the operation, the urinary catheter was removed and the second day post-operatively they started feeding. The average postoperative stay was 4.7 days, range 3 to 7 days. No patient required parenteral nutrition (Table 1).

\begin{tabular}{|l|c|l|c|c|c|c|c|c|c|c|c|c|c|}
\hline Case & $\begin{array}{c}\text { Age* } \\
\text { (Months) }\end{array}$ & Gender & $\begin{array}{c}\text { Weight } \\
\text { (kg) }\end{array}$ & $\begin{array}{c}\text { Cyst } \\
\text { type }\end{array}$ & $\begin{array}{c}\text { Cyst* } \\
\text { (cm) }\end{array}$ & $\begin{array}{c}\text { Surgical } \\
\text { date }\end{array}$ & $\begin{array}{c}\text { Total } \\
\text { time* } \\
\text { (min) }\end{array}$ & $\begin{array}{c}\text { Robot } \\
\text { Time* } \\
\text { (min) }\end{array}$ & $\begin{array}{c}\text { Bleed- } \\
\text { ing* } \\
\text { (ml) }\end{array}$ & $\begin{array}{c}\text { Conver- } \\
\text { sion }\end{array}$ & $\begin{array}{c}\text { Compli- } \\
\text { cations }\end{array}$ & $\begin{array}{c}\text { Po } \\
\text { Stay* } \\
\text { (days) }\end{array}$ & $\begin{array}{c}\text { Follow- } \\
\text { up* } \\
\text { (Months) }\end{array}$ \\
\hline 1 & 32 & Female & 15 & $1-\mathrm{A}$ & 9 & Jul-2016 & 360 & 240 & 20 & No & None & 4 & 36 \\
\hline 2 & 6 & Female & 5.9 & $1-\mathrm{A}$ & 12.5 & Aug-2016 & 344 & 184 & 40 & No & None & 7 & 35 \\
\hline 3 & 58 & Female & 19.8 & $1-\mathrm{A}$ & 9 & Aug-2017 & 382 & 262 & 50 & No & None & 3 & 23 \\
\hline 4 & 48 & Male & 19.5 & $1-\mathrm{C}$ & 8 & Sep-2018 & 364 & 234 & 20 & No & None & 5 & 22 \\
\hline
\end{tabular}

Table 1: Experience in the treatment of choledochal cyst with robotic surgery with hepaticojejunostomy Roux-en-Y in children. ${ }^{*}$ These are average value data: age 3 years, Weigth $15 \mathrm{~kg}$, Cyst size $9.6 \mathrm{~cm}$, Total time surgery $360 \mathrm{~min}$, Robot time 230 min, Bleeding 32.5 ml, PO stay 4.7 days, Follow-up 29 months. Gender 1:3 female. Roux-en-Y extracorporeal all cases. PO: postoperative. 
The smallest patient was 6 months of age, weight $5.9 \mathrm{~kg}$ and height $65 \mathrm{~cm}$, he presented the largest coledochal cyst $12.5 \mathrm{~cm}$ in greatest diameter), which occupied an important proportion of the abdomen behind the root of the mesentery, which required partial drainage by puncture, and important retroperitoneal dissection during its resection, in the postoperative period it showed clear fluid high expenditure by the active drainage, decreasing until the seventh day, when it was discharged, after drainage was withdrawn. The average postoperative follow-up was 29 months, range from 22 to 36 months, and all patients evolved asymptomatically (Table 1).

From the non-systematic review with the keywords: robotic or robot-assisted surgery + choledochal cyst + children, 22 publications were obtained, eliminating duplicates, 16 were left and of which only 10 corresponded to the subject under investigation, in addition 3 more were located different publications on the subject under investigation, resulting in 13 previous publications in total, related to the subject under investigation.

From the non-systematic review with the keywords: choledochal cyst + laparoscopic or laparoscopic surgery + children, 134 publications were obtained, and 52 publications were included, which we consider transcendent to meet the research objective, but only references with 70 or more patients, are registered in the current manuscript.

\section{Discussion}

In this study, we report our experience with robot-assisted surgery for the choledochal cyst, we treat 4 pediatric patients underwent robotic-assisted surgery for the removal of the choledochal cyst and hepaticojejunostomy, without complications, conversions, hemotransfusions and deaths. Our results coincide with what was published by Pham., et al. with the exception of surgical times, ours are more prolonged (Table 2), this study is the largest series of robot-assisted surgery for choledochal cyst in children [18].

\begin{tabular}{|c|c|c|c|c|c|c|c|c|c|}
\hline No. & Ref./year & Cases & $\begin{array}{c}\text { Age } \\
\text { (Years) }\end{array}$ & $\begin{array}{c}\text { Male/ } \\
\text { Female }\end{array}$ & $\begin{array}{c}\text { Total } \\
\text { times } \\
\text { (<min) }\end{array}$ & $\begin{array}{l}\text { Robot } \\
\text { Time* } \\
\text { (min) }\end{array}$ & $\begin{array}{c}\text { Conversion* } \\
\text { No (\%) }\end{array}$ & $\begin{array}{l}\text { Complica- } \\
\text { tions }\end{array}$ & $\begin{array}{c}\text { Po stay* } \\
\text { (days) }\end{array}$ \\
\hline 1 & Woo., et al. 2006 & 1 & 5 & $0 / 1$ & 440 & 390 & 0 & 0 & 4 \\
\hline 2 & Klein., et al. 2007 & 1 & $?$ & $?$ & $?$ & $?$ & 0 & 0 & $?$ \\
\hline 3 & Meehan., et al. 2007 & 2 & 5.5 & $1 / 1$ & 458 & 418 & 0 & 0 & 4 \\
\hline 4 & Alqahtani., et al. 2010 & 3 & $?$ & $?$ & $?$ & $?$ & 0 & 0 & $?$ \\
\hline 5 & Dawrant., et al. 2010 & 5 & 1 & $0 / 5$ & 482 & 319 & 0 & 0 & 6 \\
\hline 6 & $\begin{array}{l}\text { Akaraviputh., et al. } \\
2010\end{array}$ & 1 & 14 & $0 / 1$ & 180 & 120 & 0 & Bile leakage 1 & 20 \\
\hline 7 & Chang., et al. 2012 & 14 & 5.3 & $2 / 12$ & 570 & 324 & $1(7.1 \%)$ & $\begin{array}{c}\text { Biliary fistula } 1 \\
\text { Stenosis } 1\end{array}$ & 9 \\
\hline 8 & Wong., et al. 2013 & 1 & $?$ & $1 / 0$ & 396 & $?$ & $1(100 \%)$ & 0 & $?$ \\
\hline 9 & Alizai., et al. 2014 & 27 & 5.4 & $7 / 20$ & 479 & 302 & $5(18.5 \%)$ & One patient** & 6 \\
\hline 10 & Kim., et al. 2015 & 36 & 4.8 & $6 / 30$ & 520 & 300 & 0 & $5^{* * *}$ & 9.2 \\
\hline 11 & Zhang Zuo., et al. 2016 & 3 & 4.2 & $?$ & $?$ & 258 & 0 & 0 & 8.6 \\
\hline 12 & Wang., et al. 2018 & 1 & 7 & $0 / 1$ & 430 & 370 & 0 & 0 & 9 \\
\hline 13 & Pham., et al. 2019 & 39 & 3.4 & $9 / 30$ & 192 & 110 & 0 & 0 & 5 \\
\hline 14 & Present data & 4 & 3 & $1 / 3$ & 390 & 230 & 0 & 0 & 4.7 \\
\hline
\end{tabular}

Table 2: Previous and current series publications of robotic-assisted surgery for choledochal cyst in children. Total 138 cases.

*These are the global averages: age, total time surgery, robot time surgery, conversions and PO stay.

**Three complications: omental hernia, anastomotic stricture and subsequent bile leak.

***Three immediate complications: two minor bile leakages and one postoperative intestinal obstruction., and two 30-day complications: one stricture of the hepaticojejunostomy site and one A-loop obstruction. PO: Postoperative. 
In our cases the length of postoperative stay was the shortest (table 2) and we omitted the use of parenteral nutrition, with our protocol of early start of food in the postoperative. Recently there are publications where treatment includes the use of parenteral nutrition in patients treated with laparoscopy or with open surgery, which implies risks [13].

Previously, in our Department of Pediatric Surgery the surgery of minimal invasion of complex cases was not possible due to the limitation we face in a small number of cases, combined with the technical limitations of conventional laparoscopy and the prolonged learning curve that these pathologies require to offer efficiency and safety to our patients. To have our institution with robotic surgery technology, from the open technique we jump to minimally invasive robot-assisted surgery in complex techniques such as this one. We consider that the best option for the treatment of choledochal cyst where the technology of robotic surgery is available, is precisely the minimally invasive robot-assisted surgery.

Of our casuistry, the average size of the cysts of $9.6 \mathrm{~cm}$ can be considered important, and the larger one appeared in the smallest patient, in our opinion the size of the cyst is not a reason for conversion of a procedure, performing drainage partial cyst and this way get space in the cavity to perform the minimally invasive procedure.

Regardless of open, laparoscopic or robotic surgical approach, for the choledochal cyst, It is important to consider the following aspects in decision making and surgical technique: distal common bile duct ligation, ductoplasty for hepatic duct stenosis, intrahepatic duct and common channel protein plug clearance. Also timing of surgery for antenatally diagnosed choledochal cyst, and the Roux loop length in choldedochal cyst in children [14]. Total cyst excision should be performed as early as possible, and the optimal treatment time is the infant period [2].

The diameter of the common hepatic duct in neonates is small and the likelihood of anastomotic stricture is high. Chang., et al. propose a different way to perform hepaticojejunostomy to reduce the risk of anastomotic stenosis, an anastomosis created around the transected end of the common bile duct with the seromuscular layer of the common bile duct was sutured interruptedly to the whole layer of the jejunum [4].

In the detailed non-systematic review of previous publications in children, from the first case of choledochal cyst resection with robotic assistance that was reported in 2006, until Jul-2019, that Pham., et al. reported 39 pediatric patients, we found 13 previous publications in total, totaling 134 previous cases [15,18-29], with our patients, make a total of 138 cases of choledochal cyst treated with robotic surgery reported. The analysis of the publications provides the following: informed patients from 1 to 39 cases, average age 4.45 years, gender ratio 1: 3.85 female, average surgical times: total $408 \mathrm{~min}$ (range 180 to 570 ), console surgery time $246 \mathrm{~min}$ (range 110 to 418 ), conversions 5\% (range 0 to $100 \%$ ), complications $8.2 \%$ (range 0 to $100 \%$ ) and average postoperative stay 8.12 days (range 4 to 20 days) and $0 \%$ mortality. The above data are based on what was reported between $94.2 \%$ and $100 \%$ of the 138 cases (Table 2).

Our average surgical times of $230 \mathrm{~min}$ of console surgery, and the total average surgical time of 360 min compared to previously reported in the 134 cases, with the average surgical times: total $408 \mathrm{~min}$ and console surgery time $246 \mathrm{~min}$, are satisfactory.

In the detailed non-systematic review of previous publications in children on laparoscopic surgery in the treatment of children with choledochal cysts, from Oct-1995, when the first report was published, until Jul-2019, we include the publications that we consider relevant, which they constitute more than $90 \%$ of pediatric patients treated with choledochal cyst with conventional laparoscopic surgery, provide the following data: In total 4398 treated cases, from 52 publications, the reported cases vary from 1 to 956 , the average duration of surgery was $208 \mathrm{~min}$ (range of averages 140 to 386), data obtained in $42.7 \%$ of the total cases. The average conversion rate was $2.45 \%$ (59 cases), range 0 to $33.4 \%$, data obtained in $54.7 \%$ of the total cases. The average complication rate was $4.97 \%$ (174 cases), range 0 to $40.5 \%$, data obtained in $79.5 \%$ of the total cases. The average postoperative stay was 7 days (range 3 to 11.7), data obtained from only $31.2 \%$ of the total cases $[7,8,10,12,19,30-42]$.

Studies included in the review that compare the safety of laparoscopic operation with open surgery for choledochal cysts in children, with a large series of patients in Asian countries, with 307 patients in the open operation group and 309 patients in the laparoscopic operation group [35]. Another large multicenter study of 956 patients who underwent laparoscopic hepaticojeunostomy for choledochal cyst in seven academic institutions in the Republic of China [39], their findings suggested that laparoscopic hepaticojejunostomy represents a feasible treatment option for choledochal cyst by offering reliable middle and long-term outcome, and low surgical morbidity.

Derived from the literature review is clear that the proportion of children reported with choledochal cyst treated with robotic surgery barely reaches $3.13 \%$, compared to those treated with conventional laparoscopic surgery. Comparing the overall results between both surgical approaches: Robotic surgery, the averages rates: conversión $5 \%$, complications $8.2 \%$, and postoperative stay 8.12 days (table 2). Conventional laparoscopy, the averages rates: 
conversion $2.45 \%$, complications $4.97 \%$, and postoperative stay 7 days.

The results that we are comparing from both surgical approaches, it is logical to conclude that with conventional laparoscopic surgery there are better results than with robotic surgery for the treatment of choledochal cyst in children, however, the most important bias is the large difference in the number of patients treated with one and another surgical approach, and that the above translates into the great experience that exists with conventional laparoscopic surgery specially in the Asian countries, latitudes where the largest casuistry in the world of this pathology is presented.

Although early complications could not be avoided during the development of robotic surgical techniques (learning curve), robotic surgery for pediatric choledochal cyst showed results comparable to those for open surgery. Is considered that robotic surgery may be a valid and alternative surgery for pediatric choledochal cyst. After further development of robotic surgical systems and advancement of surgical techniques therewith, future prospective studies may reveal more positive results [28].

To date, it is also evident that very few pediatric surgeons have adopted robotic surgery in the world.

It is very important to consider that, laparoscopic choledochal cystectomy and Roux-en-Y hepaticojejunostomy requires excellent skills of operators, and the operation experience needs to be accumulated through clinical practices [13]. Diao., et al. found that the surgical time shortened when the number of cases that the operator treated exceeded 35 cases [33], this translates as the learning curve for treatment of the choledochal cyst with laparoscopy conventional.

In a Dutch National Study, they included all pediatric patients who underwent a surgical procedure for type I and IV chodedochal cyst between 1989 and 2014, were entered 91 pediatric patients underwent surgery 12 patients (13\%) laparoscopic approach, and 79 patients (87\%) open, with Roux-en-Y hepaticojejunostomy. Short-term complications of the laparoscopic approach were duodenum perforation and cholangitis, in 2 patients (16.6\%), and in five patients (42\%), ocurred cholangitis, complications requiring radiological intervention or re-surgery, stricture and incomplete of the choledochal cyst resection. They concluded that hepatobiliary laparoscopic surgery should be performed in specialized pediatric surgical centers with extensive experience in laparoscopy and hepatobiliary surgery, with exposure to a larger number of cases [43].

Recently, Zhang B., et al. report the following results with a laparoscopic approach: 7 cases converted to open surgery, 15 cases developed biliary fistula, obstruction of the jejunum loop of Roux-
en-Y in 2 cases and multiple intestinal invagination, anastomotic stenosis after hepaticojejunostomy, residual tissue of choledochal cyst and pancreatic fistula in one of each, in a series of 231 pediatric patients [10].

Reported by both groups of authors, support that conventional laparoscopy for choledochal cyst should be performed by expert surgeons and in institutions with an adequate volume of patients with this pathology.

Robotic surgery is safe and effective, technically robotic-assistance facilitates performing the biliodigestive anastomosis and for pediatric choledochal cyst showed results comparable to those for open surgery, and thus, is considered to be a valid and alternative surgery for this pathology [15,27], with the advantages of the minimum invasion. The results in the 4 cases that we treat of choledochal cyst confirm the safety and efficacy of this surgical alternative.

Analyzing some case series of choledochal cyst with conventional laparoscopy and other series of open surgery, in relation to conversions and complications, the authors report: biliary fistula is a common postoperative complication [10]. An important cause of biliary fistula is the accessory hepatic duct, their incidence is $6 \%$ to $20 \%$, and may result in bile duct infection, cholestasis and localized cirrhosis in the corresponding liver segment. This anomaly it is difficult to be detected by preoperative examination and is easy to be missed intraoperatively. In children with cholecochal cysts their recognition and treatment based on subtype of anomaly, with ductoplasty and the hepaticojejunostomy is an efficacious management [44].

Another complication is postoperative stenosis, Jung., et al. in 10/35 (28.5\%) presented postoperative biliary stricture occurred in ten patients, who underwent laparoscopic hepaticojejunostomy [45], and the preoperative hilar hepatic duct stenosis may be implicated, one large retrospective study reported that $11 \%$ of children with choledochal cyst (24/218) had bile duct stenosis at the hepatic hilum [23], and Miyano., et a.l found a higher percentage (34.5\%) of this anomaly [46].

It is also important to have the benchmark of open surgery for choledochal cyst in children, and the literature indicates that the rate of anastomotic stricture in hepaticojejunostomy with open surgery is 6 to $40 \%$ [47], while in laparoscopic surgery, it is 0.6$28.6 \%[39,40,45]$.

Bleeding is also a common complication. The causes mainly include trocar puncture injuries, injury of the gallbladder artery, hepatic artery, or portal vein, bleeding from cystic separation and abnormal coagulation [10]. In 2015, a multicenter study of exci- 
sion of laparoscopic cyst and Roux-en-Y hepaticojejunostomy for children with choledochal cysts in China, report 2 deaths, one died of hyperkalemia, and the other one died of postoperative bleeding [39]. With robotic surgery there are no reported deaths.

Based on the review of the literature and our experience, we reach the following recommendations regarding robotic hepaticojejunostomy with Roux-en-Y: 1 . The body position must be supine and reverse Trendelenburg; 2. Extracorporeal intestine-intestine anastomosis through the umbilical incision is a safe and simple method; 3. The method to expose the hilum is very simple by using the third arm of the robot, and it can also improve surgical efficiency, in total using 5 trocars.

Other techniques to expose the hilar region, are: (1) Two hitchstitch suspension technique, sutures are passed through the abdominal wall, one suspend gallbladder, and other the round ligament of the liver. (2) Liver retractor (Nathanson retractor), by an incision on the right upper abdomen, and (3) Internal suspension method: sutures for suspension round ligament of the liver, gallbladder, and stomach wall, on the internal abdominal wall $[20,24,26]$.

With the use of robotic-assisted laparoscopic surgery in the four cases of choledochal cyst in children, in our experience, the procedure was laborious, but technically and with ease the surgical anatomy was identified, the complete resection of the cyst was performed. The Roux-en-Y hepaticojejunostomy, is a process easier and more sophisticated. Our preferred technique because in our previous experience with open and now robotic approach and long-term follow-up, it is rare that they present with postoperative ascending colangitis, and on the other hand, the consequences of biliary reflux, associated with the hepaticoduodenostomy technique, are avoided.

The technical limitations of laparoscopic surgery, such as: poor ergonomics, instability of the 2D image of the surgical field with loss of depth perception, the fulcrum effect with counterintuitive movements with rigid, nonarticulated instruments, loss of handeye coordination, the presence of tremor and a prolonged learning curve, with robotic assistance are overcome [48,49].

Robotic assistance offers improvements, versus conventional laparoscopic surgery: it empowers the surgeon with his technical abilities in dissection, hemostasis and intracorporeal suture, increased articulated instrument tip dexterity, in small body spaces and anatomical sites that are difficult to access, obtaining the surgeon a shorter learning curve. It is achieved by the characteristics of robotic surgical platforms that include motion scaling, greater optical magnification, 3D and stereoscopic vision, increased articulated instrument tip dexterity, tremor filtration, operator-con- trolled camera movement, and elimination of the fulcrum effect, and all of this translates into greater efficiency and safety for patients and advantages for the surgeon. In complex and laborious techniques, less likelihood of complications and conversions [48]. Robotic assistance has special application in complex cases and reconstructive surgery, such as biliodigestive. Also, robotic surgery is suitable in the pediatric practice, which necessitates fine dissections and intracorporeal sutures in narrow anatomical spaces [50]

We believe that in hospitals where the number of cases of choledochal cyst in children is low, robotic surgery is ideal, to provide the benefits of minimal invasion effectively and safely.

Conventional laparoscopic surgery in children for choldedochal cyst should ideally be offered only by expert surgeons and in hospitals with a high volume of cases, to provide them with efficiency and safety.

If both options are not present, the recommendation is to continue with the gold standard, which is open surgery for choledochal cyst in children.

The future that awaits us is of important technological advances, so it is convenient that more pediatric surgeons in the world, start in robotic surgery for the benefit of children.

\section{Conclusion}

Our results are encouraging, and show the advantages of robotic surgery, although our experience is limited to a few cases. Robotic surgery for the treatment of choledochal cyst is feasible and safe.

The current results with both minimally invasive approaches are very similar, but we consider that it represents an important bias, the great advantage in the application that conventional laparoscopy takes to robotic surgery.

Treatment in children with choledochal cyst, nowadays it is ideal that it be with minimally invasive surgery:

- Laparoscopic: Through expert pediatric surgeons and specialized pediatric centers with a high volume of cases.

- Robot surgery: In institutions where technology is available, after pediatric surgeons have acquired the necessary skills with this approach.

If one or another situation is not present, we recommend continuing with the open approach for the surgical technique of hepaticoyeyunostomy with Roux-en-Y. To offer children the greatest safety and effectiveness.

To date, very few pediatric surgeons in the world have applied robotic surgery to the choledochal cyst. 


\section{Acknowledgements}

I thank the Pediatricians and Pediatric Surgeons of the Department of Pediatrics of the Hospital Militar de Especialidades de la Mujer y Neonatología of Mexico City, as well as, to the resident physicians of the specialty of Pediatric Surgery, for their collaboration in referring patients for treatment with robotic surgery, also all those who collaborated in the surgical teams.

\section{Conflict of Interest}

Author declares to be Proctor of the da Vinci Surgical System and sometimes receives salary for advice to Surgeons in their first robotic procedures, from the marketing company in my country, as part of the support in the training of Surgeons by this company. But, in relation to the treatment of patients and the execution of this manuscript, no economic financing was received from commercial companies.

\section{Bibliography}

1. Soares KC., et al. "Choledochal cysts: presentation, clinical differentiation, and management". Journal of the American College of Surgeons 219.6 (2014): 1167e80.

2. Bhavsar MS., et al. "Choledochal Cysts: A Review of Literature". Saudi Journal of Gastroenterology 18.4 (2012): 230-236.

3. Todani T., et al. "Classification of congenital biliary cystic disease: special reference to type Ic and IVA cysts with primary ductal stricture". Hepatobiliary Pancreatic Surgery 10 (2003): 340-344.

4. Chang X., et al. "Laparoscopic-assisted cyst excision and ductoplasty plus widened portoenterostomy for choledochal cysts with a narrow portal bile duct". Surgical Endoscopy 33 (2019): 1998-2007.

5. Lipsett PA., et al. "Choledochal cyst disease. A changing pattern of presentation". Annuals of Surgery 220.5 (1994): 644-652.

6. Olbourne NA. "Choledochal cysts. A review of the cystic anomalies of the biliary tree". Annals of the Royal College of Surgeons of England 56 (1975): 26-32.

7. Liem NT., et al. "Early and intermediate outcomes of laparoscopic surgery for choledochal cysts with 400 patients". Journal of Laparoendoscopic and Advanced Surgical Techniques A 22.6 (2012): 599-603.

8. Farello GA., et al. "Congenital choledochal cyst: video-guided laparoscopic treatment". Surgical Laparoscopy Endoscopy 5 (1995): 354-358.

9. Xu D., et al. "A modified technique of single-incision laparoscopic hepaticojejunostomy for children with choledochal cysts". BMC Surgery 19.1 (2019): 36.

10. Zhang B., et al. "Early complications after laparoscopic resection of choledochal cyst". Pediatric Surgery International 35 (2019): 845-852.
11. Shen HJ. "Laparoscopic versus open surgery in children with choledochal cysts: a meta-analysis". Pediatric Surgery International 31(2015): 529-534.

12. Lee H., et al. "Initial experience with complex laparoscopic biliary surgery in children: biliary atresia and choledochal cyst". Journal of Pediatric Surgery 39.6 (2004): 804-87.

13. Qu X., et al. "Laparoscopic Surgery in the treatment of children with Choledochal Cyst". Pakistan Journal of Medical Sciences 35.3 (2019): 807-811.

14. Yamataka A., et al. "Role of laparoscopy during surgery at the porta hepatis". South African Medical Journal 104.11 (2014): 820-824.

15. Woo R., et al. "Robot-assisted laparoscopic resection of a type I choledochal cyst in a child". Journal of Laparoendoscopic and Advanced Surgical Techniques A 16 (2006): 179-183.

16. Clavien PA., et al. "The Clavien-Dindo classification of surgical complications: five-year experience". Annuals of Surgery 250 (2009): 187-96.

17. Navarrete Arellano M and Garibay González F. "Robot-Assisted Laparoscopic and Thoracoscopic Surgery: Prospective Series of 186 Pediatric Surgeries". Frontier Pediatrics 7 (2019): 200.

18. Pham HD. "Robotic-assisted surgery for choledochal cyst in children: early experience at Vietnam National Children's Hospital". Pediatric Surgery International (2019).

19. Klein MD., et al. "Pediatric robotic surgery: lessons from a clinical experience". Journal of Laparoendoscopic and Advanced Surgical Techniques A 17.2 (2007): 265-271.

20. Meehan JJ., et al. "The robotic approach to complex hepatobiliary anomalies in children: preliminary report". Journal of Pediatric Surgery 42.12 (2007): 2110-2114.

21. Alqahtani A., et al. "Robot-assisted pediatric surgery: how far can we go?”. World Journal of Surgery 35.5 (2010): 975-978.

22. Dawrant MJ., et al. "Robot-assisted resection of choledochal cysts and hepaticojejunostomy in children less than $10 \mathrm{~kg}$ ". Journal of Pediatric Surgery 45.12 (2010): 2364-2368.

23. Akaraviputh T., et al. "Robot-assisted complete excision of choledochal cyst type I, hepaticojejunostomy and extracorporeal Roux-en-y anastomosis: a case report and review literature". World Journal of Surgery and Oncology 8.87 (2010): 1-4.

24. Chang EY., et al. "Lessons and tips from the experience of pediatric robotic choledochal cyst resection". Journal of Laparoendoscopic and Advanced Surgical Techniques A 22.6 (2012): 609-614.

25. Wong KY., et al. "Da Vinci robotic system for pediatric surgery: Report of first 20 cases". Chinese Journal of Minimally Invasive Surgery 1 (2013): 4-8. 
26. Alizai NK., et al. "Robot-assisted resection of choledochal cysts and hepaticojejunostomy in children". Pediatric Surgery International 30.3 (2014): 291-294.

27. Kim NY., et al. "Retrospective assessment of the validity of robotic surgery in comparison to open surgery for pediatric choledochal cyst". Yonsei Medical Journal 56.3 (2015): 737-743.

28. Hang Z., et al. "Da Vinci Robotic System for choledochal cysts in children". Journal of Clinical Pediatric Surgery 15.2 (2016): 137-139.

29. Wang XQ., et al. "Robotic-assisted surgery for pediatric choledochal cyst: Case report and literature review". World Journal of Clinical Cases 6.7 (2018): 143-149.

30. Liem NT., et al. "Laparoscopic complete cyst excision and hepaticoduodenostomy for choledochal cyst: early results in 74 cases". Journal of Laparoendoscopic and Advanced Surgical Techniques A 19 (2009): S87-90.

31. Nguyen Thanh L. "Laparoscopic repair for choledochal cyst: lessons learned from 190 cases". Journal of Pediatric Surgery 45.3 (2010): 540-544.

32. Diao M., et al. "A shorter loop in Roux-Y hepatojejunostomy reconstruction for choledochal cysts is equally effective: preliminary results of a prospective randomized study". Journal of Pediatric Surgery 45.4 (2010): 845-847.

33. Diao M., et al. "Laparoscopic versus Open Roux-en-Y hepatojejunostomy for children with choledochal cysts: intermediate-term follow-up results". Surgical Endoscopy 25.5 (2011): 1567-1573.

34. Diao M. "Is it necessary to ligate distal common bile duct stumps after excising choledochal cysts?". Pediatric Surgery International 27.8 (2011): 829-832.

35. Liem NT. "Is the laparoscopic operation as safe as open operation for choledochal cyst in children?". Journal of Laparoendoscopic and Advanced Surgical Techniques A 21.4 (2011): 367-370.

36. Diao M., et al. "To drain or not to drain in Roux-en-Y hepatojejunostomy for children with choledochal cysts in the laparoscopic era: a prospective randomized study". Journal of Pediatric Surgery 47.8 (2012): 1485-1489.

37. Jang JY., et al. "Laparoscopic excision of a choledochal cyst in 82 consecutive patients". Surgical Endoscopy 27.5(2013): $1648-1652$

38. Diao M., et al. "Single-incision versus conventional laparoscopic cyst excision and Roux-Y hepaticojejunostomy for children with choledochal cysts: a case-control study". World Journal of Surgery 37.7 (2013): 1707-1713.

39. Qiao G., et al. "Laparoscopic cyst excision and Roux-Y hepaticojejunostomy for children with choledochal cysts in China: a multicenter study". Surgical Endoscopy 29.1 (2015): 140-144.
40. Senthilnathan P., et al. "Laparoscopic Management of Choledochal Cyst-Technical Modifications and Outcome Analysis". World Journal of Surgery 39.10 (2015): 2550-2556.

41. Yu BH and Lin F. "Clinical effects in resection of congenital choledochal cyst of children and jejunum Roux-Y anastomosis by laparoscope". European Review for Medical and Pharmacological Sciences 20.21 (2016): 4530-4534.

42. Song G., et al. "Comparative clinical study of laparoscopic and open surgery in children with choledochal cysts". Saudi Medical Journal 38.5 (2017): 476-481.

43. van den Eijnden MHA, et al. "Choledochal Malformation in Children: Lessons Learned from a Dutch National Study". World Journal of Surgery 41 (2017): 2631-2637.

44. Diao M., et al. "Laparoscopic management for aberrant hepatic duct in children with choledochal cysts". Surgical Endoscopy 33.7 (2019): 2376-2380.

45. Jung K., et al. "Is preoperative subclassification of type I choledochal cyst necessary?". Korean Journal of Radiology 13 (2012): S112-S116.

46. Miyano G., et al. "Comparison of laparoscopic hepaticojejunostomy and open hepaticojejunostomy. Can stenosis of the hilar hepatic duct affect postoperative outcome?". Asian Journal of Endoscopic Surgery 10.3 (2017): 295-300.

47. Kim JH., et al. "Risk factors of postoperative anastomotic stricture after excision of choledochal cysts with hepaticojejunostomy". Journal of Gastrointestinal Surgery 12.5 (2008): 822-828.

48. Cundy TP., et al. "The first decade of robotic surgery in children". Journal of Pediatric Surgery 48.4 (2013): 858-865.

49. Koga $\mathrm{H}$ and Yamataka A. "Current status and future of pediatric endoscopy surgery". Nihon Geka Gakkai Zasshi 116.5 (2015): 320-322.

50. Chaussy Y., et al. "Robot-assisted surgery: current status evaluation in abdominal and urological pediatric surgery". Journal of Laparoendoscopic and Advanced Surgical Techniques A 23 (2013): 530-538.

\section{Volume 2 Issue 11 November 2019 (C) All rights are reserved by Mario Navarrete Arellano.}

